\title{
Características clínicas e prevalência de sintomas depressivos em pacientes com a doença de Parkinson
}

\author{
Alisson Menezes Araújo Lima*; Fabiana de Campos Cordeiro Hirata**; Ione Cavalcante Lacerda***; \\ Rosa Maria Salani Mota****; Veralice Meireles Sales de Bruin ${ }^{* * * *}$
}

\section{Resumo}

A doença de Parkinson (DP) é uma afecção caracterizada por acinesia, rigidez e tremor de repouso. Flutuações clínicas são comumente observadas e não são bem compreendidas; provavelmente, guardam relação com o estado de neurotransmissão dopaminérgica e com influências de outros neurotransmissores. O objetivo do trabalho foi avaliar as características clínicas, hábitos de vida e presença de sintomas depressivos na DP por meio do Inventário de Depressão de Beck (IDB). Quarenta pacientes consecutivos, sendo 27 homens $(67,5 \%)$ com idade entre 48 e 84 anos $(63,2 \pm$ 8,5), com Hoehn e Yahr entre I e III, foram avaliados. Na amostra estudada, 28 casos $(70 \%)$ apresentavam sintomas depressivos (IDB > 10).

Palavras-chave: Depressão. Doença de Parkinson. Perfil epidemiológico.

\section{Introdução}

O histórico da doença de Parkinson (DP) teve sua primeira descrição em 1817, quando James Parkinson, médico inglês, publicou a monografia "An essay on the shaking palsy", na qual descrevia as principais manifestações da doença. $\mathrm{Na}$ época, a encefalite era a causa mais comum do parkinsonismo, síndrome caracterizada por tremor de repouso, rigidez, acinesia e instabilidade postural. Alguns anos depois, o neurologista francês Jean Martin Charcot propôs a denominação de DP em homenagem a James Parkinson e descreveu duas formas polares da doença: uma forma rígida-acinética e a outra com predomínio de tremor. Relatou também a disautonomia e alterações secundárias, dentre as quais a disartria e a micrografia. (TEIVE, 1998).

* Mestre em Ciências Farmacêuticas pela Universidade Federal do Ceará. Endereço para correspondência: Av. Senador Fernandes Távora, 101, apt. 116, bloco D, Bairro Jóquei Clube, CEP 60510-290, Fortaleza - CE. E-mail: alissonlima@pmenos.com.br; alisson_farma@yahoo.com.br

** Acadêmica do curso de Medicina da Universid̄ade Federal do Ceará.

***: Acadêmica do curso de Enfermagem da Universidade Estadual de Ceará.

***** Mestra em Estatística pela Universidade de São Paulo. Professora Adjunto IV da Universidade Federal do Ceará, Campus do PICI, Departamento de Estatística.

****** Doutora em Psicobiologia pela Universidade Federal de São Paulo. Professora de Neurologia da Faculdade de Medicina da Universidade Federal do Ceará.

$\longrightarrow$ Recebido em fevereiro de 2009 - Avaliado em março de 2009.

$\rightarrow$ doi:10.5335/rbceh.2009.026 
Atualmente, sabe-se que a DP é uma doença neurodegenerativa e progressiva que acomete o sistema nigroestriatal dopaminérgico (OBESO et al., 2000), clinicamente caracterizada por distúrbios do movimento, da postura e do equilíbrio, manifestando-se através de acinesia, rigidez, tremor de repouso e instabilidade postural. As características clínicas e a fisiopatogênese da DP foram reconhecidas desde o início do século XX, porém a etiologia da DP permanece desconhecida. (SMITH; KIEVAL, 2000).

As doenças degenerativas do sistema nervoso que comumente se associam ao envelhecimento, como a DP, têm se tornado de importância crescente, dado que nos últimos anos observa-se um aumento da expectativa de vida da população. $\mathrm{O}$ custo associado à terapia medicamentosa e com cuidadores tem levado à necessidade de um maior número de estudos relacionados a esse assunto.

No continente norte-americano aproximadamente $1 \%$ da população acima de 65 anos é afetada pela doença, valor que corresponde a cerca de um milhão de pessoas. Observa-se uma prevalência de aproximadamente 160 casos para cem mil habitantes e uma incidência de vinte novos casos para cada 100.00 habitantes nesse mesmo continente. (RAJPUT, 1992).

Uma recente projeção feita por cientistas da Universidade de Rochester, nos Estados Unidos prevê um número duas vezes maior de pacientes com DP até o ano de 2030. Nesse estudo, no qual foram avaliados os 15 maiores países do mundo, relatou-se que o número de parkinsonianos deve passar dos atuais 4,1 milhões de pacientes com DP para aproximadamente 8,7 milhões de pessoas acometidas pela doença, o que demonstra o grau de importância dos estudos relacionados a esta alteração patológica. (DORSEY et al., 2007).

As manifestações clássicas da DP, que constituem a chamada "síndrome parkinsoniana", são acinesia, rigidez e tremor. Situações de estresse emocional ou a sensação de ser observado aumentam sensivelmente a intensidade do tremor. Esses sintomas, geralmente, se iniciam com características assimétricas, acometendo um braço e gradualmente evoluindo para outras extremidades. Com o avançar da doença, ocorre redução da expressão facial e do piscar de olhos, redução do volume da voz, perda assimétrica do balanço dos braços durante a marcha, fadiga e lentificação dos movimentos. Outras alterações, tais como disfunção cognitiva, depressão, alterações gastrointestinais e sintomas autonômicos, podem, em graus variáveis, fazer parte do quadro. (RINNE, 1990). Vale ressaltar que os sinais e sintomas de natureza motora são os mais comuns e que, na maioria dos casos, a função intelectual e a capacidade de julgamento estão preservadas na DP.

Sintomas depressivos podem acometer entre $4 \%$ a $70 \%$. A taxa mais baixa foi encontrada num estudo inicial no qual não havia escalas objetivas padronizadas disponíveis, enquanto nos estudos mais recentes a taxa relatada variou entre $25 \%$ a $70 \%$. Na DP, os sintomas depressivos podem se manifestar como tristeza, falta de apetite, alteração do peso, fadiga, distúrbios do sono, redução da autoestima, ansiedade e pensamentos suicidas. Diversos autores sugerem 
que os sintomas depressivos na DP são, provavelmente, diferentes dos observados em outras condições clínicas associadas à depressão. Embora o humor depressivo observado na DP tenha sido considerado por alguns como uma consequência natural a um estado de doença progressiva e devastadora, há evidências que sugerem que esta manifestação decorre, primariamente, de alterações neurobiológicas. (CUMMINGS, 1992).

O Inventário de Depressão de Beck é uma escala de autoavaliação amplamente utilizada para avaliar a intensidade do transtorno depressivo por possuir um questionário de 21 itens, incluindo atitude, sintomas depressivos e ideação suicida, cuja intensidade é indicada por uma pontuação que varia de 0 a 3 . A avaliação deste inventário se dá com a soma dos escores, onde um resultado superior a 10 pontos designa a presença de sintomas depressivos. (GORESTEIN; ANDRADE, 1998).

Os pacientes com doença de Parkinson apresentam complicações relacionadas tanto à evolução da doença quanto ao uso de agentes medicamentosos. No presente trabalho, analisamos as características clínicas, hábitos de vida e presença de sintomas depressivos em pacientes com DP.

\section{Métodos}

Trata-se de um estudo de experimentação clínica, multidisciplinar, utilizando um grupo de quarenta pacientes consecutivos, com diagnóstico clínico de DP e acompanhados no ambulatório de Distúrbios do Movimento do Hospital
Universitário da Faculdade de Medicina da Universidade Federal do Ceará. Os pacientes foram avaliados quanto ao perfil socioeconômico e à presença de sintomas depressivos.

A amostra foi composta por quarenta pacientes com diagnóstico clínico de doença de Parkinson. Foram envolvidos aqueles com Hoehn e Yahr (H-Y) entre I e III (HOEHN; YAHR, 1967) por apresentarem incapacidades de leve a moderada. Todos os casos estavam em uso de levodopa, tendo obtido resposta terapêutica.

O termo de consentimento livre e esclarecido foi fornecido por meio de uma declaração padrão, escrita em linguagem acessível. Cada paciente, antes de assinar o documento, leu e analisou a declaração. Nenhum paciente foi incluído no estudo antes da obtenção de seu consentimento livre e esclarecido.

Os pacientes foram orientados a ingerir a dose matinal estável de levodopa por volta de $8 \mathrm{~h}$, com uma tolerância de 30min. Em seguida os questionários foram iniciados. Como parâmetros de identificação, avaliaram-se a idade dos indivíduos no início do estudo e o sexo (masculino ou feminino). Em relação à história clínica, observou-se a duração da doença, avaliando o período decorrido em anos desde o diagnóstico até a época atual.

Optou-se pelo Inventário de Depressão de Beck, que é uma medida de autoavaliação de sintomas depressivos amplamente utilizada tanto em pesquisa como em clínica, tendo sido traduzida para vários idiomas e validada em diferentes países. Há várias propostas de diferentes pontos de corte para distinguir os níveis 
de sintomas depressivos utilizando o IDB. De acordo com Beck et al. (1988), a escolha do ponto de corte adequado depende da natureza da amostra e dos objetivos do estudo. Para amostras de pacientes com transtorno afetivo o Center for Cognitive Therapy recomenda os seguintes pontos de corte: menor que 10 = sem depressão ou depressão mínima; de 10 a 18 = depressão, de leve a moderada; de 19 a 29 = depressão, de moderada a grave; de 30 a $63=$ depressão grave. Assim, optou-se pelo ponto de corte em 10 pontos, pois acredita-se ser uma boa taxa de especificidade, acurácia e correlação para a amostra, como já comprovado em outros estudos. (OLIVEIRA et al., 2006).

Os dados foram submetidos a um pacote do programa de estatística SPSS for Windows, versão 12.0.

\section{Resultados}

Estudaram-se quarenta pacientes com diagnóstico clínico de DP com idade entre 48 e 84 anos $(63,20 \pm 8,55)$, sendo 27 do sexo masculino $(67,5 \%)$ e 13 do feminino $(32,5 \%)$. Conforme o critério de inclusão previamente esclarecido e definido, a Escala de Hoehn \& Yahr, que avalia a gravidade da doença, oscilou entre os estágios I e III. A idade de apresentação dos sintomas oscilou entre 41 e 80 anos $(56,80 \pm 9,41)$. A duração total da doença variou entre 1 a 25 anos $(6,55 \pm 5,03)$. Todos os casos utilizavam levodopa e, quanto à dose, observou-se que a mínima catalogada foi de $150 \mathrm{mg}$ e a máxima, $1.250 \mathrm{mg} /$ dia $(653,75 \pm 283,1)$. Os pacientes estudados apresentaram média de índice de massa corpórea (IMC) de 24,5 ( $\pm 2,5)$, variando entre 19,8 a 32. Os dados demográficos relativos à população estudada estão representados na Tabela 1.

Tabela 1 - Características clínicas da amostra com diagnóstico clínico de doença de Parkinson $(\mathrm{n}=40)$.

\begin{tabular}{l|r}
\hline \multicolumn{1}{c|}{ Dados gerais } & \multicolumn{1}{c}{ Resultados } \\
\hline \multicolumn{1}{c|}{$\mathrm{N}$} & 40 \\
\hline Sexo (M/F) & $27 / 13$ \\
Percentagem M/F (\%) & $67,5 / 32,5$ \\
Variação da idade (anos) & $48-84$ \\
$\quad$ Média \pm desvio-padrão & $63,20 \pm 8,55$ \\
Variação da idade de início da doença (anos) & $41-80$ \\
$\quad$ Média \pm desvio-padrão & $56,80 \pm 9,41$ \\
Variação da duração da doença (anos) & $1-25$ \\
$\quad$ Média \pm desvio-padrão & $6,55 \pm 5,03$ \\
Variação da dose de levodopa (mg/dia) & $150-1250$ \\
$\quad$ Média \pm desvio-padrão & $653,75 \pm 283,1$ \\
Variação do índice de massa corpórea & $19,8-32,0$ \\
$\quad$ Média \pm desvio-padrão & $24,5 \pm 2,54$ \\
\hline
\end{tabular}

Nota: $\mathrm{M}=$ masculino; $\mathrm{F}$ = feminino. 
Dezoito pacientes $(45 \%)$ relataram ingestão frequente de bebidas alcoólicas, e o hábito de fumar estava presente em 14 casos (35\%). História familiar de DP foi relatada em $40 \%(n=16)$ dos casos. Os dados relativos a hábitos de vida e histórico familiar da população estudada estão representados na Tabela 2.

Tabela 2 - Hábitos de vida e histórico familiar de DP na população em estudo.

\begin{tabular}{lcc}
\hline & Dados gerais & Resultados Sim/Não (\%) \\
\hline Álcool (S/N) & $45 / 55$ \\
Fumo (S/N) & $35 / 65$ \\
Histórico familiar de DP (\%) (S/N) & $40 / 60$ \\
\hline
\end{tabular}

$\mathrm{DP}=$ Doença de Parkinson.

O Inventário de Depressão de Beck mostrou uma variação de 0 a 29 pontos, com média de 12,98 $( \pm 7,74)$ pontos.
Vinte e oito pacientes (70\%) apresentavam sintomas depressivos $(\mathrm{BDI}>10)$ (Tab. 3).

Tabela 3 - Resultados da presença de sintomas depressivos (inventário de Beck) na amostra.

\begin{tabular}{l|c}
\hline \multicolumn{1}{c|}{ Avaliações } & Total \\
\hline Variação do Inventário de Depressão de Beck & $0-29$ \\
Média \pm desvio-padrão & $12,98 \pm 7,74$ \\
Sintomas depressivos & $70 / 30$ \\
\hline
\end{tabular}

\section{Discussão}

O estudo envolveu, predominantemente, homens e com idade média de 63,2 anos, sendo esta série semelhante a outras séries de DP na literatura que mostram um predomínio do sexo masculino a partir da sexta década. (WERNECK; ALVARENGA, 1999).

A média diária de levodopa observada foi de $653,8 \pm 283,1$ e está bem próxima a outros estudos feito na mesma região, que tiveram média de 625 \pm 233 ,0. (MEDEIROS, 2005). Vale ressaltar que os pacientes apresentavam gravidade da doença entre os estágios I e III na Escala de Hoehn e Yahr, o que pode explicar parcialmente os baixos níveis de levodopa utilizados. Outro fator a ser considerado é a dificuldade de acesso ao medicamento por razões sociais e/ou financeiras.

Utilizou-se o IMC para avaliação do estado nutricional e diagnóstico de sobrepeso e obesidade, observando-se uma variação no resultado de 19,8 a 32. O uso do IMC em idosos apresenta dificuldades em razão do decréscimo de estatura, acúmulo de tecido adiposo, redução da massa corporal magra e diminuição da quantidade de água no organismo, não havendo, assim, um ponto de corte específico para essa faixa etária. Diante dessas dificuldades, utilizou-se a classificação sugerida pela OMS para idosos: desnutridos (IMC < 22), eutróficos (IMC entre 22 e 27) e obesos (IMC > 27). (DUARTE; REGO, 2007). De acordo com essa classificação, no presente estudo 28 casos (70\%) foram conside- 
rados eutróficos; $7(17,5 \%)$, obesos e 5 $(12,5 \%)$, desnutridos. Estudos recentes têm também demonstrado associação entre o IMC e o risco de desenvolver a DP, pois alguns pacientes portadores de DP desenvolvem com frequência disfagia, que geralmente pode se associar à perda de peso. O paciente com DP tenta se adaptar às dificuldades e pode não procurar atenção médica em virtude da disfagia e/ou desnutrição. (KNOOP; PADOVANI, 2001).

Neste estudo observou-se história familiar de DP em 16 pacientes, correspondendo a $40 \%$ da amostra total. Tal achado é superior ao descrito na literatura, que mostra uma variação entre 14,5\% (WERNECK; ALVARENGA, 1999) e $27 \%$ dos casos. (RIBEIRO et al., 2004). É possível que essa discordância seja secundária ao fato de se tratar de uma amostra pequena. Vale ressaltar que tem sido sugerido que a influência do fator genético é complexa e, provavelmente, depende de fatores circunstanciais e da influência de outros mecanismos.

O hábito de consumo de bebida alcoólica de forma não compulsiva antes do aparecimento dos sintomas foi relatado em 18 pacientes (45\% da amostra). Vários estudos investigam a associação entre o uso de determinadas substâncias e o aparecimento da DP na tentativa de encontrar uma relação etiológica. (RIBEIRO et al., 2004).

O álcool é um agente sabidamente tóxico para o sistema nervoso. Anteriormente, estudos sobre este assunto mostraram um possível caráter protetor tanto do álcool quanto do café e do tabaco. Observou-se o hábito de fumar em 14 pacientes (35\% da amostra). Estudos sugerem um caráter protetor do fumo para o surgimento da DP. (BENEDETTI et al., 2000; WERNECK; ALVARENGA, 1999). $\mathrm{Na}$ amostra de quarenta pacientes deste estudo o hábito de fumar foi relatado com certa frequência, sugerindo que o fumo não desempenhou um papel protetor.

Sintomas depressivos estavam presentes em 28 pacientes $(70 \%)$ da amostra. A literatura relata, em média, $40 \%$ dos pacientes com sintomas depressivos. (PRADO; BARBOSA, 2005). Tal achado pode variar entre 4 a $70 \%$, dependendo da metodologia aplicada. (SILBERMAN et al., 2004). A depressão antecede os sintomas motores em $25 \%$ dos pacientes com DP. Geralmente, a depressão na DP está associada com o avanço da gravidade da doença, corroborada pela alta pontuação na escala UPDRS (Unified Parkinson Disease Rating Scale) e estágio avançado na escala de H-Y.

\section{Conclusão}

Neste estudo os pacientes eram, predominantemente, do sexo masculino e tinham idade superior a cinquenta anos, estando de acordo com a maioria das séries de DP na literatura.

Hábitos de ingerir bebida alcoólica e/ou fumar estavam presentes em porcentagens consideráveis, o que não indica caráter protetor dessas substâncias. Observou-se a presença de sintomas depressivos em $70 \%$ dos casos, que é um dado preocupante, pois há tendência de piora nos sintomas da doença e na qualidade de vida do paciente. 
Clinical characteristics and predominance of depressive symptoms in patients with the Parkinson's disease

\section{Abstract}

Parkinson's disease (PD) is a chronic illness characterized by the presence of akinesia, rigidity and resting tremor. Clinical fluctuation is a common finding and is probably related to the oscillation of dopaminergic neurotransmission and also to the influence of other neurotransmitters. The aim of this study was to it evaluate the clinical characteristics, habits of life and presence of depressive symptoms on a population with PD with the Beck Depression Inventory (BDI). All cases were out patients from a movement disorders clinic. Forty consecutive patients, 27 male $(67,5 \%)$, age from 48 to 84 years $(63,2 \pm 8,5)$, Hoehn e Yahr between I e III were included. Twentyeight cases presented depressive symptoms (BDI > 10).

Key words: Depression. Health Profile. Parkinson disease.

\section{Referências}

BECK, A. T.; STEER, R. A; GARBIN, M. G. Psychometric properties of the Beck Depression Inventory: twenty-five years of evaluation. Clinical Psychology Review, v. 8, n. 1, p. 77-100, 1988.

BENEDETTI, M. D. et al. Smoking, alcohol and coffee consumption preceding Parkinson's disease. Neurology, v. 55, n. 9, p. 1350-1358, 2000.

CUMMINGS, J. L. Depression and Parkinson's disease: a review. Am. J. Psychiatry., v. 149 , n. 5 , p. 443-454, 1992.

DORSEY, E. R. et al. Projected number of people with Parkinson disease in the most populous nations, 2005 through 2030. Neurology, v. 68, n. 5, p. 384-386, 2007.
DUARTE M. B.; REGO M. A. V. Comorbidade entre depressão e doenças clínicas em um ambulatório de geriatria. Cadernos de Saúde Pública, Rio de Janeiro, v. 23, n. 3, p. 691-700, 2007.

GORESTEIN, C.; ANDRADE, L. Inventário de depressão de Beck: propriedades psicométricas da versão em português. Revista de Psiquiatria Clínica, Itatiba, v. 25, n. 5, p. 245-250, 1998.

HOEHN, M. M.; YAHR, M. D. Parkinsonism: onset, progression and mortality. Neurology, v. 17, n. 5 , p. 427-442, 1967.

KNOOP, D.; PADOVANI, M. Voz, fala e deglutição. In: LIMONGI, J. C. P. (Org.). Conhecendo melhor a doença de Parkinson. São Paulo: Plexus, 2001. p. 117-135.

MEDEIROS C. A. M. Avaliação sobre os efeitos da melatonina na disfunção motora e no sono na doença de parkinson: um estudo randomizado, duplo-cego e controlado com placebo. 2005. Dissertação (Mestrado em Ciências Farmacêuticas) - Universidade Federal do Ceará, Fortaleza, 2005.

OBESO J. A. et al. Pathology of the basal ganglia in Parkinson's disease. Trends in Neurosciences, v. 23, n. 1, p. 8-19, 2000.

OLIVEIRA, K. L. et al. Relação entre ansiedade, depressão e desesperança entre grupos de idosos. Psicologia em Estudo, Maringá, v. 11, n. 2, p. 351-359, 2006.

PRADO, R. C. P.; BARBOSA, E. R. Depression in Parkinson's disease. Arq. Neuropsiquiatr, v. 63, n. 3b, p. 766-771, 2005.

RAJPUT, A. H. Frequency and cause of Parkinson's disease. Can. J. Neurol. Sci., v. 19, n. 1, p. 103-107, 1992.

RIBEIRO, E. M. et al. Bases genéticas da doença de Parkinson. Revista Brasileira de Medicina, São Paulo, v. 61, n. 6, p. 388-398, 2004.

RINNE, U. K. Early dopamine agonist treatment in Parkinson's disease. Adv. Neurol. v. 53, p. 417-419, 1990. 
SILBERMAN, C. D. et al. Uma revisão sobre depressão como fator de risco na doença de Parkinson e seu impacto na cognição. Revista de Psiquiatria, Rio Grande do Sul, v. 26, n. 1, p. 52-60, 2004.

SMITH, Y.; KIEVAL, J. Z. Anatomy of the dopamine system in the basal ganglia. TINS, v. 23, n. 10 , p. $28-33,2000$.

TEIVE, H. A. G. O papel de Charcot na doença de Parkinson. Arquivos de Neuropsquiatria, São Paulo, v. 56, n. 1, p. 141-145, 1998.

WERNECK, A. L. S.; ALVARENGA, H. Genetics, drugs and environmental factors in Parkinson's disease: a case-control study. Arquivos de Neuropsquiatria, São Paulo, v. 57, n. 2b, p. 347-355, 1999. 